\title{
MHD FLOW OF NANO-FLUID WITH NON-UNIFORM HEAT SOURCE OR SINK IN THE PRESENCE OF CHEMICAL REACTION AND ACTIVATION ENERGY
}

\author{
Akindele Akintayo Oladimeji ${ }^{1 *}$, Ogunsola Amos Wale ${ }^{2}$, Adebowale Martins Obalalu ${ }^{3}$, Adegbite peter ${ }^{4}$, Ajala \\ Olusegun Adebayo ${ }^{5}$, Saheed Alao ${ }^{6}$ \\ *1,2,4,5,6 Department of Pure and Applied Mathematics, Ladoke Akintola University of Technology, Ogbomoso, Oyo State \\ Nigeria. \\ ${ }^{3}$ Department of Statistics and Mathematical sciences, Kwara State University, Malete, Kwara State, Nigeria.
}

*Corresponding Author: -

E-mail address: aoakindele65@pgshcool.lautech.edu.ng

\begin{abstract}
: -
The study of squeezing flow cannot be over emphasized due to its numerous applications in manufacturing industries, food processing, lubricating system, power transmission and medical equipment are few of such processes. This calls for more studies in the area of squeezing flow. MHD flow of nano-fluid with non-uniform heat source or sink in the presence of chemical reaction and activation energy were considered. The governing partial differential equations were transformed to ordinary differential equations in terms of suitable similarity variables together with the initial and boundary conditions. The resulting equations were then solved using Newton's finite difference technique with the aid of MAPLE 18.0 software. The effect of radiation, magnetic parameter, temperature dependent heat source parameter, surface dependent heat source parameter and other associated physical parameters on the flow system were reported.
\end{abstract}

Keywords: heat source or sink; porosity; activation energy; brownian motion; thermophoresis motion; chemical reaction; magneto hydrodynamic.

\section{(c) $($ ) (1)}




\section{INTRODUCTION}

The need for an improved understanding of heat and mass transfer phenomena in squeezing flow of fluids between two parallel surfaces cannot be over emphasized due to its numerous applications and importance in Applied Sciences and many Engineering fields, such as soil mechanics, powder metallurgy, chemical processing and petroleum reservoir recovery processes as can be found in Bergman et al. [1].

In the past efforts to analyze squeezing flow of fluids between two parallel surfaces, Sobamowo et al. [2] presented thermo-magneto-solutal squeezing flow of nanofluid between two parallel disks embedded in a porous medium where the effects of nanoparticles geometry slip and temperature jump conditions were reported. Sravan and Rushi [3] examine the effect of homogeneous-heterogeneous reactions in MHD stagnation point nanofluid flow toward a cylinder with nonuniform heat source or sink. Hayat et al. [4] studied the unsteady flow of nanofluid through porous medium with variable characteristics. Recently, Muhammad [5] considered serious solutions for unsteady axisymmetric flow over a rotating stretchable disk with deceleration. The numerical study of heat transfer and viscous flow in a dual rotating extendable disk system with a non-Fourier heat flux model was reported by Shamshuddin et al. [6]. Hayat et al. [7] explored a flow between two stretchable rotating disks with Cattaneo-Christov heat flux model. MHD fluid flow and heat transfer due to stretchable rotating disk was reported by Mustafa [8]. Akindele and Ogunsola [9] worked on the study of non-isothermal permeable flow of nano-fluids in a stretchable rotating disk system. Recent research about squeezing flow of fluids between two parallel surfaces can be mentioned by the studies [10-20]. Vimal et al. [21] studied numerical investigation of magnetic nanofluids flow over rotating disk embedded in a porous medium. Abubakar et al. [22] considered the stability analysis on the flow and heat transfer of nanofluid past a stretching/shrinking cylinder with suction effect. Fang and Zhang [23] reported the flow between two stretchable disks with exact solution of Navier-Stokes equations. Mushtaq and Mustafa [24] discussed the computations for nanofluid flow near a stretchable rotating disk with axial magnetic field and convective conditions. The research of Mustafa [25] explains nanofluid flow and heat transfer due to a rotating disk. Entropy generation in bioconvection nanofluid flow between two stretchable rotating disks was carried out by Khon et al. [26]. Abubakar et al. [27] inquired the stability analysis on the flow and heat transfer of nanofluid past a stretchable/ shrinking cylinder with suction effect.

The objective of the present study is to analyze the development of the unsteady MHD flow of nanofluid with non-uniform heat source or sink in the presence of chemical reaction and activation energy in a cylindrical coordinate. The radial and tangential velocity profiles, thermal and concentration profiles are taken into consideration.

\section{MATHEMATICAL FORMULATION}

Consider the unsteady three-dimensional squeezing flow of nanofluid in a stretchable rotating disks system shown in Fig.1. Magnetic force takes place between the two disks and the upper disk is maintained at temperature $T_{2}$ while the lower disk is at a lower temperature $\mathrm{T}_{1}$, also Darcy's law is not negligible. Hence, the time dependent angular velocity is $\Omega_{1}(t)=\frac{\Omega_{1}}{1-b t}$ follows:

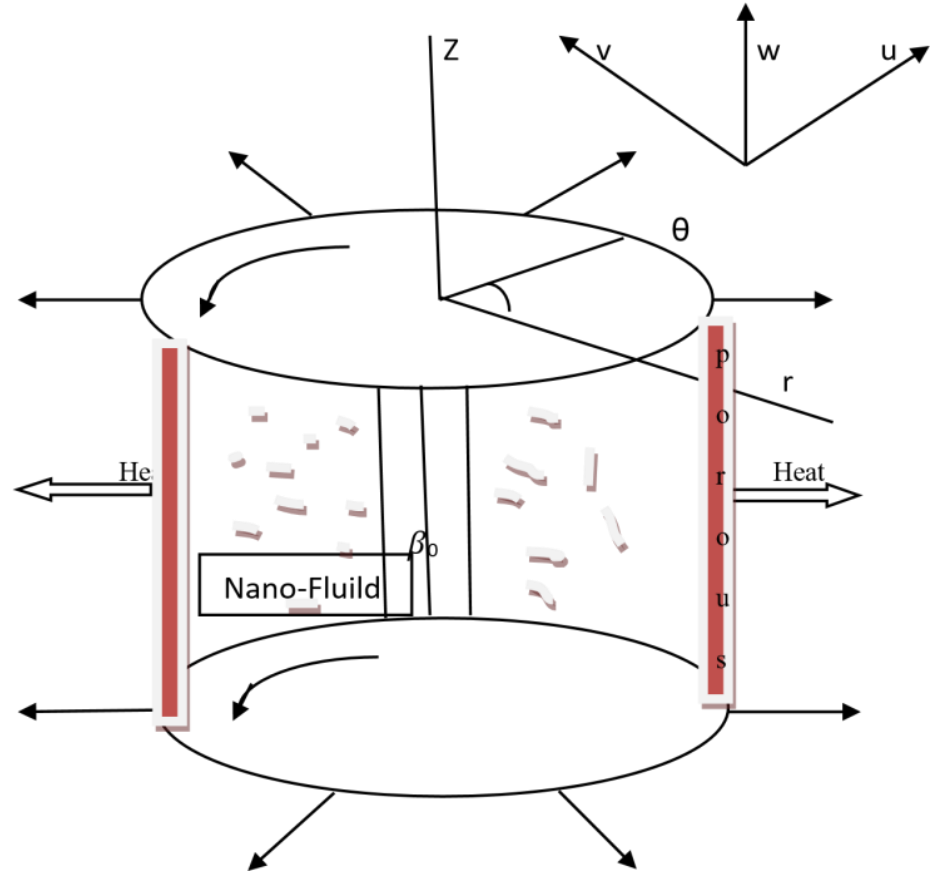

Fig.1: squeezing flow of nanofluid between two rotating disks with permeable wall 


$$
\begin{aligned}
& \frac{\partial u}{\partial r}+\frac{u}{r}+\frac{\partial w}{\partial z}=0 \\
& \frac{\partial u}{\partial t}+u \frac{\partial u}{\partial r}+w \frac{\partial u}{\partial z}-\frac{v^{2}}{r}=-\frac{1}{\rho} \frac{\partial p}{\partial r}+v\left(\frac{\partial^{2} u}{\partial z^{2}}\right)-\frac{v \varepsilon(z) u}{k(z)}-\frac{C b \varepsilon^{2}(z) u \sqrt{u^{2}-v^{2}}}{\sqrt{k(z)}}-\frac{\sigma_{0} \beta_{0}^{2} u}{\rho} \\
& \frac{\partial v}{\partial t}+u \frac{\partial v}{\partial r}+w \frac{\partial v}{\partial z}+\frac{u v}{r}=v\left(\frac{\partial^{2} v}{\partial z^{2}}\right)-\frac{v \varepsilon(z) v}{k(z)}-\frac{C b \varepsilon^{2}(z) v \sqrt{u^{2}-v^{2}}}{\sqrt{k(z)}}-\frac{\sigma_{0} \beta_{0}^{2} v}{\rho} \\
& \frac{\partial T}{\partial t}+u \frac{\partial T}{\partial r}+w \frac{\partial T}{\partial z}=\frac{K}{\rho C_{p}}\left(\frac{\partial^{2} T}{\partial z^{2}}\right)+\frac{\varepsilon v}{\sigma}\left(D_{B} \frac{\partial T}{\partial z} \frac{\partial C}{\partial z}+\frac{D_{T}}{T_{\infty}}\left(\frac{\partial T}{\partial z}\right)^{2}\right)-\frac{1}{\rho C_{p}} \frac{\partial q_{r}}{\partial z}+q^{\prime \prime \prime} \\
& \frac{\partial C}{\partial t}+u \frac{\partial C}{\partial r}+w \frac{\partial C}{\partial z}=D_{B}\left(\frac{\partial^{2} C}{\partial z^{2}}\right)+\frac{D_{T}}{T_{\infty}}\left(\frac{\partial^{2} T}{\partial z^{2}}\right)-K r^{2}\left(C-C_{w}\right)\left(\frac{T}{T_{\infty}}\right)^{m} \exp \left(\frac{-E a}{k_{0} T}\right)
\end{aligned}
$$

with initial and boundary conditions

$$
\begin{aligned}
& u=\frac{a r}{(1-b t)}, \quad v=\frac{r \Omega_{1}}{(1-b t)}, \mathrm{w}=0, \mathrm{~T}=\mathrm{T}_{\mathrm{w}}, \mathrm{C}=\mathrm{C}_{\mathrm{w}} \quad \text { at } \mathrm{z}=0 \\
& \mathrm{u}=0, v=0, \mathrm{w}=0, \mathrm{~T}=\mathrm{T}_{\mathrm{w}}, \mathrm{C}=\mathrm{C}_{\infty} \quad \text { at } \mathrm{z} \rightarrow \infty
\end{aligned}
$$

where

$$
\begin{aligned}
& k(z)=k_{\infty}\left(1+d \exp ^{-\frac{z}{\gamma}}\right), \varepsilon(z)=\varepsilon_{\infty}\left(1+j \exp ^{\frac{-z}{\gamma}}\right) \\
& q^{\prime \prime \prime}=\frac{k \Omega_{1}}{\rho C_{p}(1-b t) \nu}\left[Q\left(T-T_{\infty}\right)+Q_{1}\left(T_{w}-T_{\infty}\right) \exp ^{-\eta}\right]
\end{aligned}
$$

\section{SIMILARITY TRANSFORMATIONS}

Following [4], similarity transformation is invoked:

$$
\begin{aligned}
& u=\frac{\Omega_{1} r}{(1-b t)} f^{\prime}(\eta), v=\frac{\Omega_{1} r}{(1-b t)} g(\eta), w=-2 \sqrt{\frac{\Omega_{1} v}{1-b t}} f(\eta), \quad \eta=z \sqrt{\frac{\Omega_{1}}{v(1-b t)}} \\
& \theta(\eta)=\frac{T-T_{\infty}}{T_{w}-T_{\infty}}, P=\frac{\rho v \Omega_{1}}{(1-b t)} P(\eta), \quad \phi(\eta)=\frac{C-C_{\infty}}{C_{w}-C_{\infty}}
\end{aligned}
$$

The Rosseland relation for radiative heat flux qr is [21].

$$
q_{r}=-\frac{4 \sigma *}{3 k *} \frac{\partial T^{4}}{\partial z},
$$

where $\sigma^{*}$ denotes the Stefan-Boltzmann constant and $\mathrm{k}^{*}$ shows the mean absorption coefficient. We assume that the difference in the temperature in the flow analysis is such that the term $\mathrm{T}^{4}$ may be expanded in a Taylor series. Hence, expanding $\mathrm{T}^{4}$ about $\mathrm{T}_{\infty}$ and omitting higher terms we obtain

$$
T^{4} \cong 4 T_{\infty} T-3 T_{\infty}^{4}
$$

hence, substituting equation (11) into equation (10), we have

$$
q_{r}=-\frac{16 \sigma * T_{\infty}^{3}}{3 k *} \frac{\partial T}{\partial z}
$$

where, $u, v, w$ are the radial, tangential, and axial velocity components in the $(\mathrm{r}, \theta, \mathrm{z})$ directions respectively. $T$ is the temperature, $v$ is the kinematic viscosity, $p$ is hydrodynamic pressure of the fluid and $\rho$ is the density of the fluid.

The mass conservation law equation (1) is identically satisfied. However, velocity equations, energy and the concentration equations are reduced to the equivalent nonlinear coupled system of ordinary differential equations: 


$$
\begin{aligned}
& f^{\prime \prime \prime}+2 f f^{\prime \prime}-f^{\prime 2}+g^{2}-S\left(f^{\prime}+\frac{\eta f^{\prime \prime}}{2}\right)-\frac{1}{\alpha \operatorname{Re}}\left(\frac{1+j \exp ^{-\eta}}{1+d \exp ^{-\eta}}\right) f^{\prime}-\beta\left(\frac{\left(1+j \exp ^{-\eta}\right)^{2}}{\sqrt{1+d \exp ^{-\eta}}}\right)\left(f^{\prime 2}+\frac{g^{2}}{2}\right) \\
& -H a f^{\prime}=0 \\
& g^{\prime \prime}+2 f g^{\prime}-2 f^{\prime} g-S\left(g+\frac{\eta g^{\prime}}{2}\right)-\frac{1}{\alpha \operatorname{Re}}\left(\frac{1+j \exp ^{-\eta}}{1+d \exp ^{-\eta}}\right) g-\beta\left(\frac{\left(1+j \exp ^{-\eta}\right)^{2}}{\sqrt{1+d \exp ^{-\eta}}}\right)\left(\frac{f^{\prime 2}}{2}+g^{2}\right)-H a g=0 \\
& (1+R) \theta^{\prime \prime}+2 \operatorname{Pr} f \theta^{\prime}+\operatorname{Pr}\left(N_{b} \theta^{\prime} \varphi^{\prime}+N_{t} \theta^{\prime 2}-\frac{S \eta}{2} \theta^{\prime}\right)+\left(Q \theta+Q_{1} \exp ^{-\eta}\right)=0 \\
& \varphi^{\prime \prime}+\frac{N_{t}}{N_{b}} \theta^{\prime \prime}+2 L e f \varphi^{\prime}-\frac{S \eta L e \varphi}{2}-\operatorname{Le} \delta\left(1+\delta_{1} \theta\right)^{m} \exp ^{\left[\frac{-E}{1+\delta_{1} \theta}\right]} \varphi=0
\end{aligned}
$$

The corresponding boundary conditions (7) were transformed to:

$$
\begin{aligned}
& f(0)=0, f^{\prime}(0)=A, g(0)=1, \theta(0)=1, \varphi(0)=1 \\
& f^{\prime}(\infty)=0, g(\infty)=0, \theta(\infty)=0, \varphi(\infty)=0
\end{aligned}
$$

Where ( Re) denotes Reynolds number, $(\operatorname{Pr})$ prandtl number, $(\mathrm{S})$ and $(\mathrm{A})$ are the unsteadiness parameters, $(\alpha)$ the porosity parameter, $(\mathrm{E})$ is the activation energy parameter, $(\delta)$ the reaction rate parameter, $\left(\delta_{1}\right)$ the temperature difference parameter, $(\mathrm{Ha})$ is the hartman number, $(\mathrm{R})$ radiation parameter, $(\mathrm{m})$ exponential constant, $(\mathrm{Q})$ the temperature dependent heat source parameter, $\left(\mathrm{Q}_{1}\right)$ the surface dependent heat source parameter, $\left(\beta^{*}\right)$ local inertial parameter, Le is lewis number, $\left(\mathrm{N}_{\mathrm{t}}\right)$ the thermophoresis parameter and $\left(\mathrm{N}_{\mathrm{b}}\right)$ Brownian motion parameter, and then:

$$
\left.\begin{array}{l}
\operatorname{Re}=\frac{U_{W} r}{v}, \beta^{*}=\frac{C b \varepsilon_{\infty}^{2} r}{\sqrt{k_{\infty}}}, L e=\frac{v}{D_{B}}, \operatorname{Pr}=\frac{\rho c_{p} v}{K}, N_{b}=\frac{\varepsilon D_{B}\left(C_{1}-C_{2}\right)}{\sigma}, \delta=k_{0}^{2}\left(\frac{1-b t}{\Omega_{1}}\right), \\
N_{t}=\frac{\varepsilon D_{T}\left(T_{1}-T_{2}\right)}{T_{\infty} \sigma}, S=\frac{b}{\Omega_{1}}, A=\frac{a}{\Omega_{1}}, H a=\frac{\sigma_{0} \beta_{0}^{2}}{\rho h}, R=\frac{16 \sigma * T_{\infty}^{3}}{3 k * K}, E=\frac{E a}{k_{0} T_{\infty}}, \alpha=\frac{k_{\infty}}{r^{2} \varepsilon_{\infty}}, \\
\delta_{1}=\frac{T_{w}-T_{\infty}}{T_{\infty}}
\end{array}\right\}
$$

\section{RESULTS AND DISCUSSION}

Equations (13) to (16) along with the associated boundary conditions (17) were solved numerically via Newton's finite difference method with the help of Maple 18.0 software. In the investigation, we analyze MHD flow of nano-fluid with non-uniform heat source or sink in the presence of chemical reaction and activation energy, the effect of flow parameters were obtained from the solution. The purpose of this section is to interpret the graphical description of sundry variables such as local Reynolds number $(\operatorname{Re})$, Prandtl number $(\operatorname{Pr})$, porosity parameter $(\alpha)$, local inertial parameter $\left(\beta^{*}\right)$, reaction rate parameter $(\delta)$, temperature difference parameter $\left(\delta_{1}\right)$, unsteadiness parameters $(\mathrm{S}, \mathrm{A})$, Hartman number (magnetic parameter) $(\mathrm{Ha})$, radiation parameter $(\mathrm{R})$, temperature dependent heat source parameter $(\mathrm{Q})$, surface dependent heat source parameter $\left(Q_{1}\right)$, variable permeability parameter $(d)$, variable porosity parameter $(j)$, Lewis number (Le), Brownian motion parameter $\left(\mathrm{N}_{\mathrm{b}}\right)$, Thermophoresis parameter $\left(\mathrm{N}_{\mathrm{t}}\right)$, constant of exponential parameter $(\mathrm{m})$ and activation energy parameter $(\mathrm{E})$ on radial velocity $f^{\prime}(\eta)$, tangential velocity $g(\eta)$, thermal field $\theta(\eta)$ and the concentration field $\varphi(\eta)$. Fig. 2 displayed the behavior of unsteadiness parameter (S) on radial velocity. Higher (S) leads to a stronger radial velocity profile. It was found in Fig.3 that increment in the porosity parameter $(\alpha)$ leads to a higher radial velocity profile. Fig.4 elaborate role of Reynolds number $(\mathrm{Re})$ on radial velocity. It shows an increment in $(\mathrm{Re})$ leads to an higher radial velocity profile. Increment in the radial velocity profile was witnessed on Fig. 5 through the increase in the variable permeability parameter (d) while Fig.6 analyzed that higher estimation of the variable porosity parameter (j) lowers the radial velocity profile. Characteristics of local inertial parameter $\left(\beta^{*}\right)$ and unsteadiness parameter (A) on radial velocity profile are portrayed in Fig.7 and Fig.8 respectively. $\left(\beta^{*}\right)$ reduces the profile while (A) enhance the profile. Fig.9 and Fig.10 are sketched to scrutinize the behavior of tangential velocity and thermal field through $(\mathrm{S})$ respectively. Enhancement in the tangential velocity profile is observed through higher $(S)$ and reduction in the thermal field is noted through higher (S). Fig. 11 displayed the behavior of $\left(\mathrm{N}_{\mathrm{b}}\right)$ on the thermal field. Higher $\left(\mathrm{N}_{\mathrm{b}}\right)$ leads to stronger thermal field. Fig.12 and Fig.13 shows the impact of increasing magnetic field parameter $(\mathrm{Ha})$ on the radial and tangential velocities respectively, (Ha) is noticeably reduced on both profiles. The effect of $(\mathrm{Q})$ and $\left(\mathrm{Q}_{1}\right)$ are demonstrated on Fig.14 and Fig.15 respectively. It was found that both $(\mathrm{Q})$ and $\left(\mathrm{Q}_{1}\right)$ enhance the thermal field. Fig.16, it was found that a increase in radiation parameter (R) enhance the absorbing rate $\left(\mathrm{k}^{*}\right)$ which decrease the temperature profile. Fig.17 displayed the behavior of activation energy parameter $(\mathrm{E})$ on the concentration profile. Higher $(\mathrm{E})$ leads to stronger concentration profile and more associated layer thickness. Fig.18 and Fig.19 witnessed the effect of $(\mathrm{m})$ and $(\delta)$ on the concentration profile respectively. It was observed that both $(\mathrm{m})$ and $(\delta)$ reduce the profile. Table. 1 shows the comparison result of local Nusselt number reported by Hayat et al.[4] and the present work. Table.2 illustrated the behavior of radial skin friction, tangential skin friction, local Nusselt number and local Sherwood number for various flow parameters. 
Table.1 Comparison table for values of $\left(\mathrm{Re}_{\mathrm{r}}\right)^{1 / 2} \mathrm{Nu}$ obtained for $\mathrm{Q}=\mathrm{Q} 1=\mathrm{R}=\mathrm{Ha}=\mathbf{0}$

\begin{tabular}{lccccc}
$\mathbf{S}$ & $\mathbf{N}_{\mathbf{b}}$ & $\mathbf{N t}$ & $\mathbf{P r}$ & Hayat et al. $[4]$ & Present work \\
\hline 0.0 & 0.2 & 0.3 & 0.8 & 0.23835 & 0.23321 \\
\hline
\end{tabular}

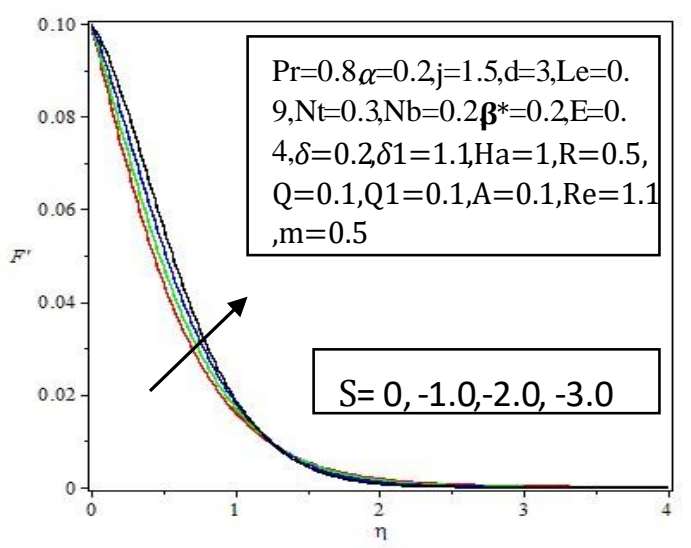

Fig.2. Behavior of $f^{\prime}(\Pi)$ against $S$.

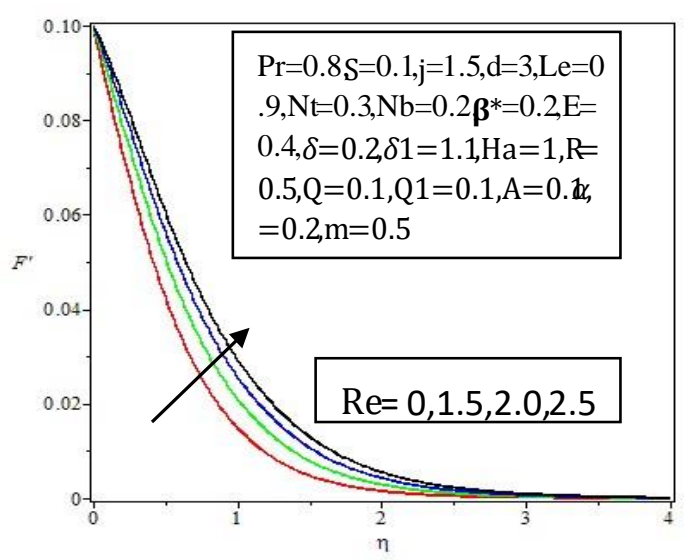

Fig.4. Behavior of $f^{\prime}(\eta)$ against Re.

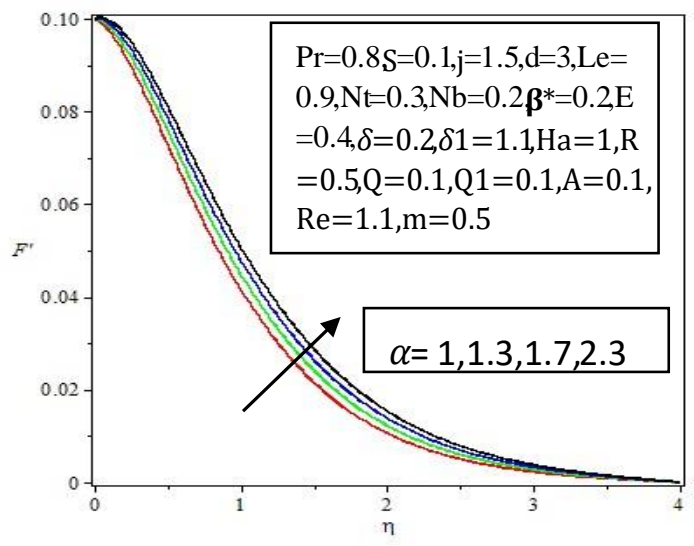

Fig.3. Behavior of f ' $(\square)$ against $\alpha$.

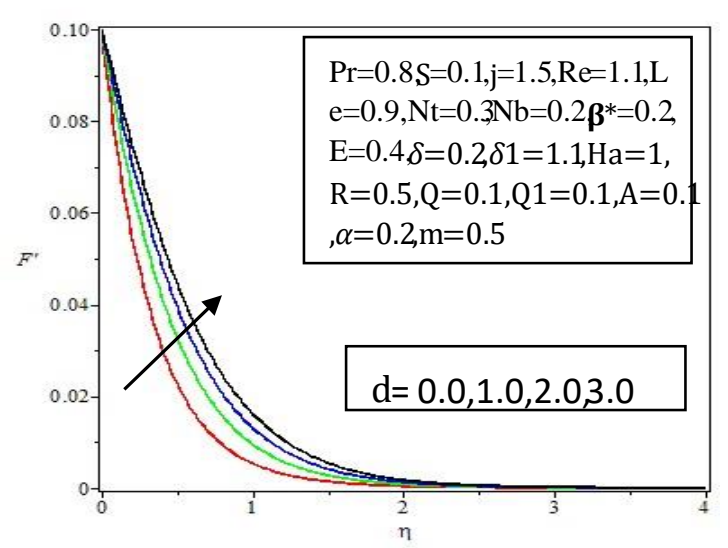

Fig.5. Behavior of $f^{\prime}(\eta)$ against $d$. 


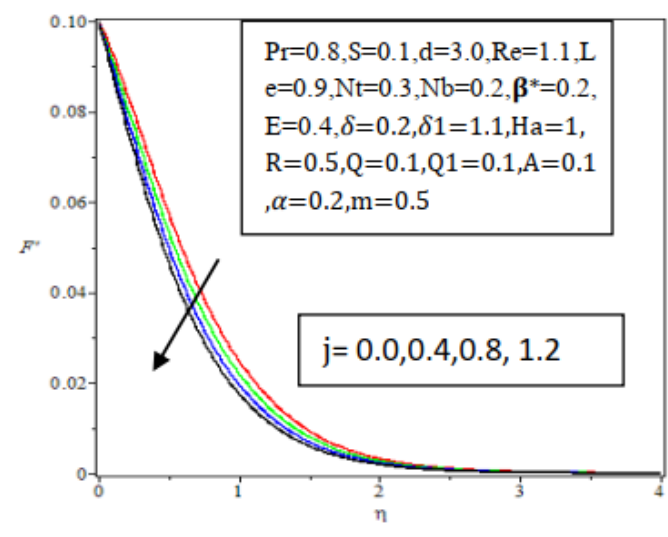

Fig.6. Behavior of $\mathrm{f}^{\prime}(\eta)$ against $\mathrm{j}$.

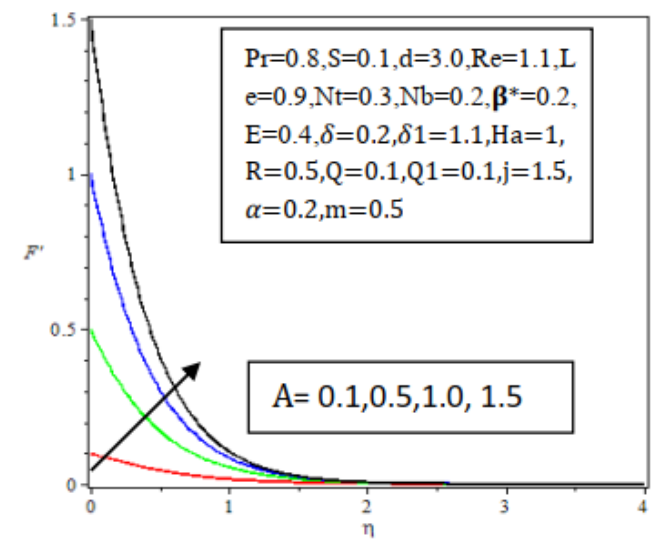

Fig.8. Behavior of $\mathrm{f}^{\prime}(\eta)$ against $A$.

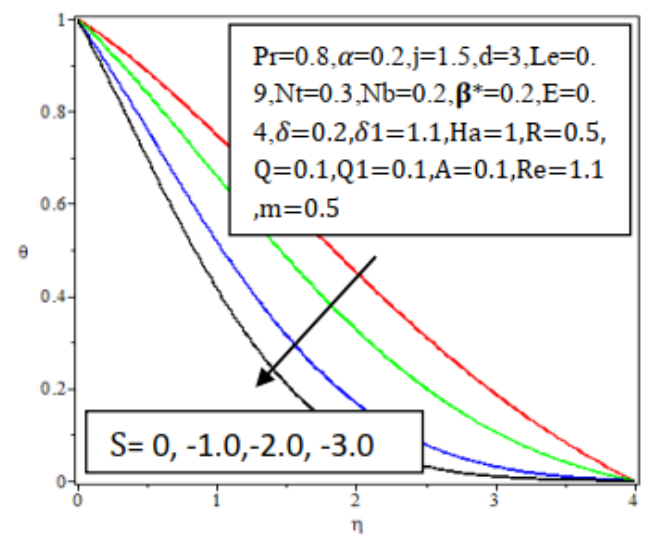

Fig.10. Behavior of $\theta(\eta)$ against $S$.

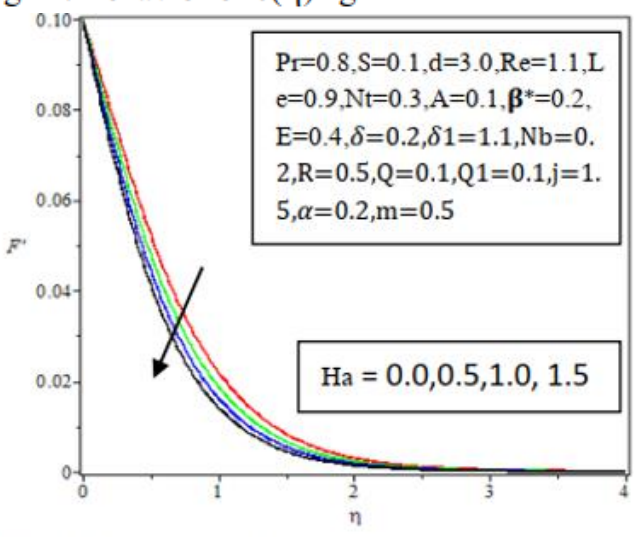

Fig.12. Behavior of $\mathrm{f}^{\prime}(\eta)$ against Ha.

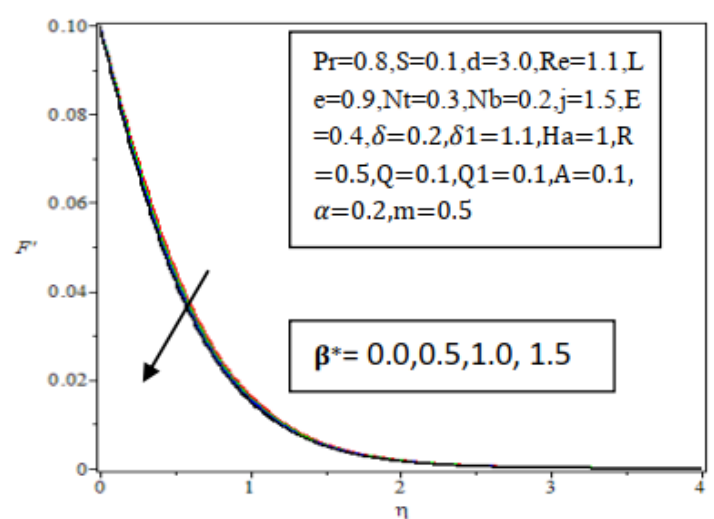

Fig.7. Behavior of $f^{\prime}(\eta)$ against $\boldsymbol{\beta}^{*}$.

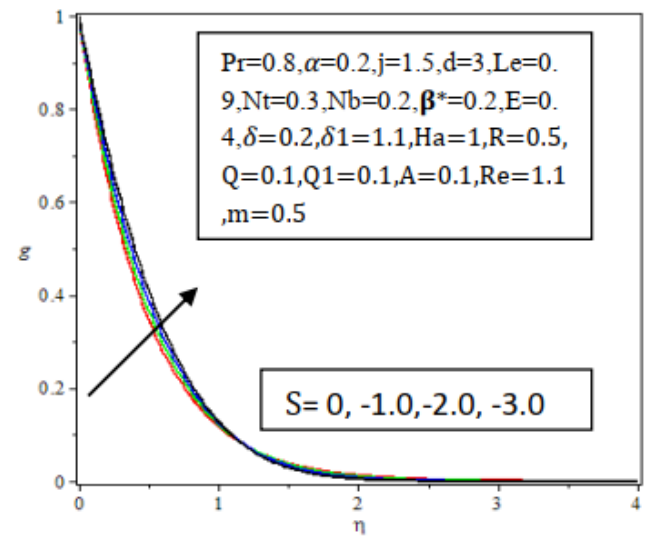

Fig.9. Behavior of $g(\eta)$ against $S$.

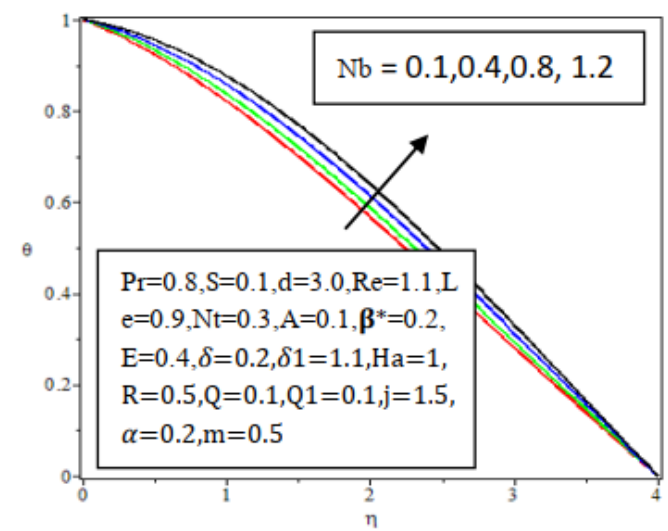

Fig.11. Behavior of $\theta(\eta)$ against $\mathrm{Nb}$.

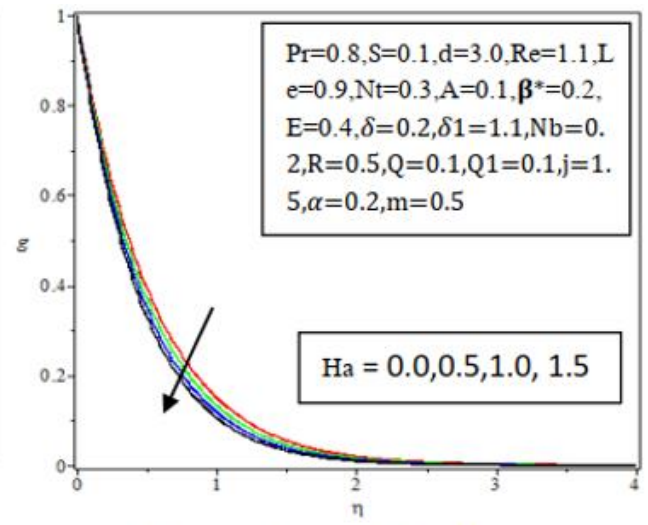

Fig.13. Behavior of $g(\eta)$ against Ha. 


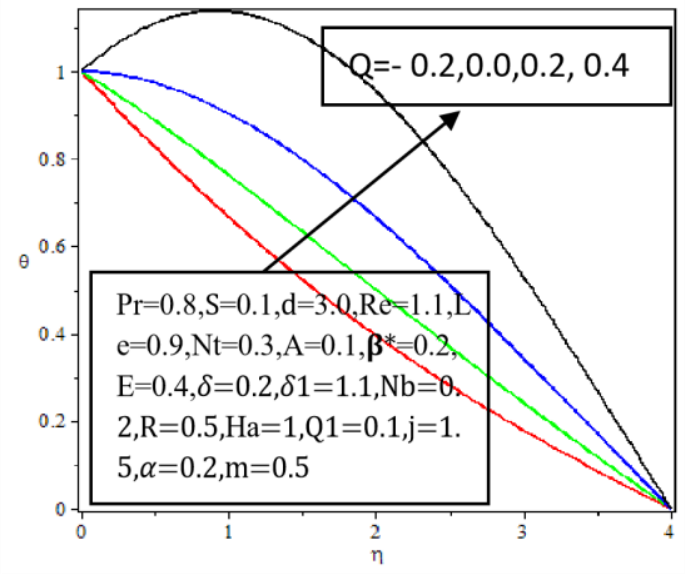

Fig.14. Behavior of $\theta(\eta)$ against $Q$.

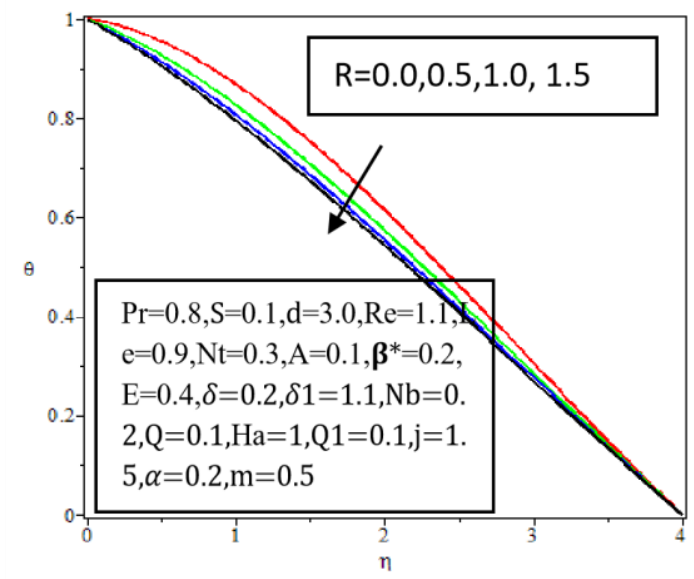

Fig.16. Behavior of $\theta(\eta)$ against $\mathrm{R}$.

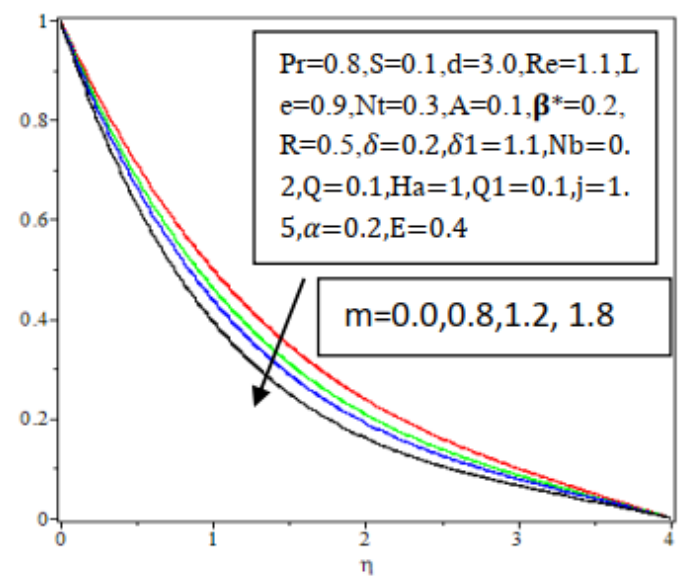

Fig.18. Behavior of $\varphi(\eta)$ against $m$.

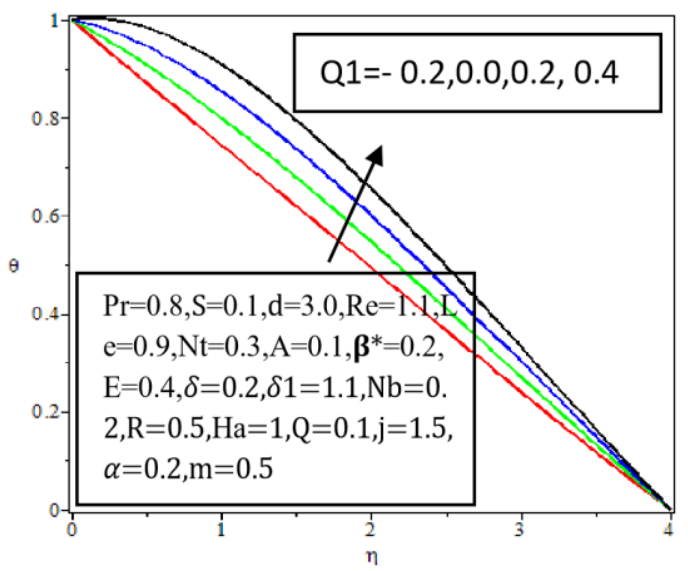

Fig.15. Behavior of $\theta(\eta)$ against Q1.

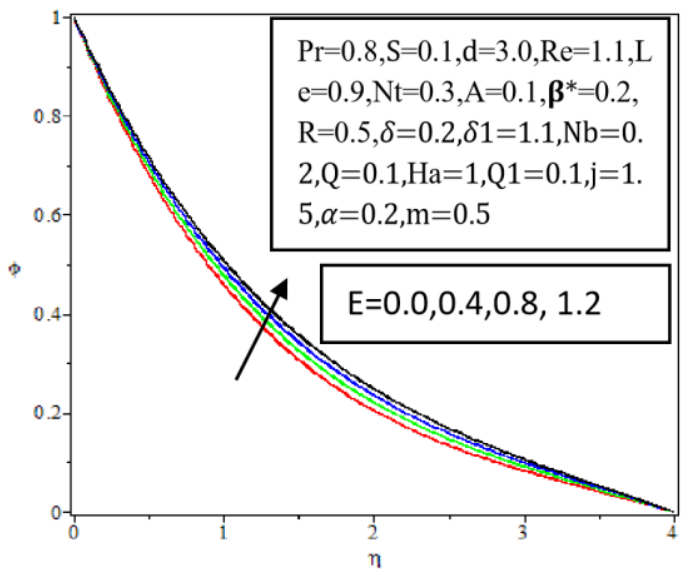

Fig.17. Behavior of $\varphi(\eta)$ against E.

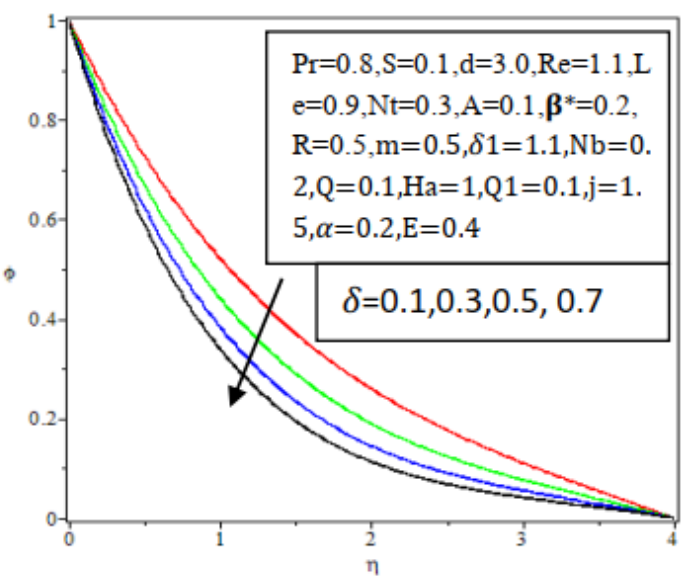

Fig.19. Behavior of $\varphi(\eta)$ against $\delta$.

Table 2: Radial skin friction coefficient, tangential skin friction coefficient, local Nusselt number and local sherwood number for different parameters.

\begin{tabular}{llllllllll}
\hline $\mathrm{S}$ & $\sigma$ & $\mathrm{Ha}$ & $\mathrm{R}$ & $\mathrm{Q}$ & $\mathrm{Q} 1$ & $-\left(\mathrm{Re}_{\mathrm{r}}\right)^{1 / 2} \mathrm{Cf}$ & $-\left(\mathrm{Re}_{\mathrm{r}}\right)^{1 / 2} \mathrm{Cg}$ & $-\left(\mathrm{Re}_{\mathrm{r}}\right)^{1 / 2} \mathrm{Nu}$ & $-\left(\mathrm{Re}_{\mathrm{r}}\right)^{1 / 2} \mathrm{Sh}^{2}$ \\
\hline 0.0 & 0.2 & 1.0 & 0.5 & 0.1 & 0.1 & 0.123933 & 2.132276 & 0.093071 & 0.532107 \\
-1.0 & - & - & - & - & - & 0.098566 & 1.953453 & 0.287590 & 0.487659 \\
-2.0 & - & - & - & - & - & 0.070632 & 1.763728 & 0.446253 & 0.532169 \\
0.0 & 1.1 & - & - & - & - & 0.012779 & 1.466879 & 0.107925 & 0.520448 \\
-1.0 & - & - & - & - & - & -0.041138 & 1.202171 & 0.305718 & 0.492205 \\
-2.0 & - & - & - & - & - & -0.107259 & 0.912376 & 0.464852 & 0.550562 \\
0.0 & 1.2 & - & - & - & - & 0.009637 & 1.451576 & 0.108522 & 0.519696 \\
-1.0 & - & - & - & - & - & -0.045632 & 1.184037 & 0.306489 & 0.492755 \\
-2.0 & - & - & - & - & - & -0.113546 & 0.891181 & 0.465655 & 0.551697 \\
\hline
\end{tabular}




\section{CONCLUSION}

In this paper, the effect of radiation parameter, temperature dependent heat source parameter, surface dependent heat source parameter and magnetic parameter on the squeezing nanofluid flow in a stretchable rotating disk system have been investigated using the Newton's finite differential method. Also, the influence of various flow parameters such as Brownian motion parameter, thermophoresis parameter, Lewis number, Prandtl number, Raynolds number, local inertial parameter, unsteadiness parameter, activation energy parameter, reaction rate parameter and porosity parameter on the radial and tangential velocity components, temperature and concentration profiles were investigated. It is confirmed in this work that the radiation parameter enhance the adsorption rate which decreases the temperature profile. Base on the work the following remarks were made.

1) As the unsteadiness parameter increase, the radial and tangential velocities component increases significantly while thermal field decrease.

2) The radial and tangential velocities component decelerates significantly with an increase in the magnetic parameter.

3) Increasing the temperature dependent heat source parameter and the surface dependent heat source parameter evokes a corresponding increase in the thermal field for both parameters.

4) Increasing the value of the activation energy, the concentration distribution increases while the constant of exponential reduced the concentration profile.

\section{CONFLICT OF INTERESTS}

The author(s) declare that there is no conflict of interests.

Nomenclature

$(\mathrm{r}, \theta, \mathrm{z})$ : Cylindrical coordinate

$\mathrm{u}, \mathrm{v}, \mathrm{w}$ : velocity component

A: Unsteadiness parameter

b: Unsteadiness constant

Cb: Drag coefficient

C: Concentration $\left(\mathrm{kg} / \mathrm{m}^{3}\right)$

$\mathrm{C}_{\mathrm{w}}$ : Surface concentration

$\mathrm{C}_{\infty}$ : Ambient concentration

$\mathrm{C}_{\mathrm{p}}$ : $\quad$ Specific heat at constant pressure $(\mathrm{J} / \mathrm{kg} \mathrm{K})$

$\mathrm{Cf}, \mathrm{Cg}$ : Skin friction coefficients

$\mathrm{d}$ : variable permeability

$\mathrm{D}_{\mathrm{B}}$ : Brownian motion coefficient $\left(\mathrm{m}^{2} / \mathrm{s}\right)$

$\mathrm{D}_{\mathrm{T}}$ : Thermophoretic diffusion coefficient $\left(\mathrm{m}^{2} / \mathrm{s}\right)$

E: Activation energy parameter

Ea: Activation energy

f: Radial velocity profile

g: Tangential velocity profile

Ha: Hartmann number (magnetic parameter)

$\mathrm{j}: \quad$ variable porosity

$\mathrm{K}$ : Thermal conductivity

$\mathrm{k}_{0}$ : Boltzmann constant

$\mathrm{kr}$ : Chemical reaction constant

$\mathrm{k}^{*}$ : Absorption coefficient

$\mathrm{k}_{\infty}$ : permeability

Le: Lewis number

$\mathrm{m}$ : Exponential constant

$\mathrm{N}_{\mathrm{b}}$ : Brownian motion parameter

$\mathrm{N}_{\mathrm{t}}$ : Thermoporesis parameter

$\mathrm{Nu}$ : Nusselt number

P: Pressure $\left(\mathrm{N} / \mathrm{m}^{2}\right)$

Pr : Prandtl's number

Q: Temperature dependent heat source or sink

$\mathrm{Q}_{1}$ : Surface dependent heat source or sink

$\mathrm{Re}_{\mathrm{r}}$ : Local Reynolds number

S: Unsteadiness parameter

Sh: Sherwood number

$\mathrm{t}$ : Time

T: Fluid temperature

$\mathrm{T}_{\mathrm{w}}$ : Surface temperature

$\mathrm{T}_{\infty}$ : Ambient temperature

$\mathrm{u}$ : Radial velocity

v: Tangential velocity

w: Axial velocity 


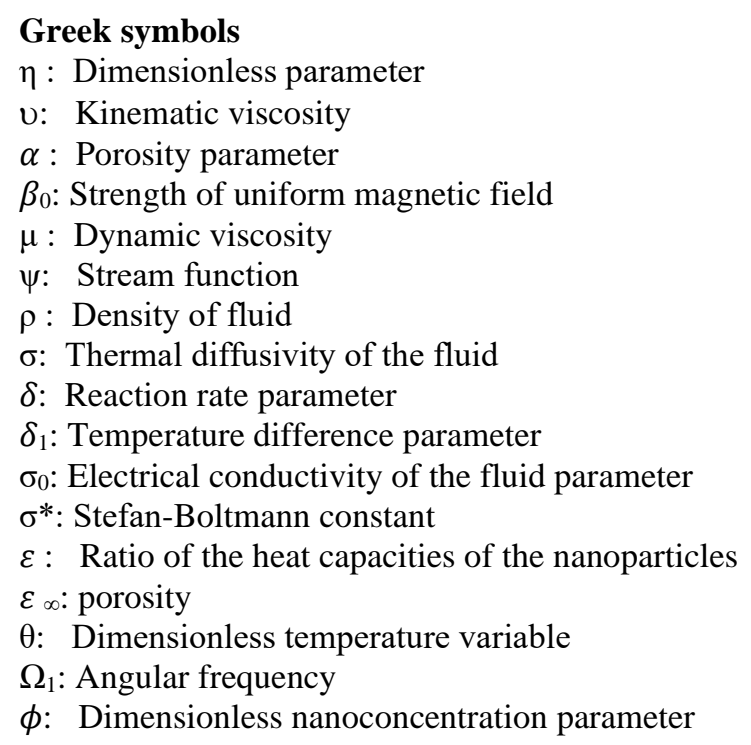

\section{REFERENCES}

[1].Bergman, Lavine, Incropera and Dewitt, (2011): Introduction to Heat Transfer (sixth edition), wiley.

[2].Sobamowo, M.G., Akinshilo, A.T., Yinusa, A.A.: (2018) Thermo-Magneto-Solutal squeezing flow of nanofluid between two Parallel disk embedded in a porous medium: Effects of nanoparticule geometry, slip and temperature jump conditions. Modelling and Simulation in Engineering, Volume 2018, Articule ID 7364634, 18 pages https://doi.org/10.1155/2018/7364634

[3].Sravan Kumar, T and Rushi Kumar, B.: (2019) Effect of homogeneous-heterogeneous reactions in MHD stagnation point nanofluid flow toward a cylinder with nonuniform heat source or sink. Applied Mathematics and Computing, Trends in Mathematics, https://doi.org/10.1007/978-3-030-01123-9_29

[4].Hayat Tasawar., Haider Farwa., Alsaedi Ahmed., Ahmad Bashir.: (2020) Unsteady flow of nanofluid through porous medium with variable characteristics. International Communications in heat and mass transfer 119(2020) 104904, https://doi.org/10.1016/j.icheatmasstransfer.2020/104904

[5].Muhammad Adil Sadiq.: (2020) Serious solution for unsteady axisymmetic flow over a rotating stretchable disk with deceleration. Symmetry2020, 12,96

[6].Shamshuddin, M.D., Mishra, S.R., Anwar Beg, O., Ali Kadir.: (2018) Numerical study of heat transfer and viscous flow in a dual rotating extendable disk system with a non-Fourier heat flux model. Wiley Periodicals Inc: Heat transfer- Asian Res. 2019;48:435-459 DOI: 10.1002/htj.21392

[7].Hayat Tasawar., Qayyum Sumaira., Imtiaz Maria., Alsaedi Ahmed.: (2017) Flow between two stretchable rotating disks with Cattaneo-christov heat flux model. Results in physics 7 (2017) 126-133 http://dx.doi.org/10.1016/j.rinp.2016.12.007

[8].Mustafa Turkyilmazoglu.: (2012) MHD fluid flow and heat transfer due to a stretching rotating disk. International journal of thermal sciences 51.195-201

[9].Akindele Akintayo Oladimeji.,Ogunsola Amos Wale.: (2021) A study of non-isothermal permeable flow of nanofluid in a stretchable rotating disk system. J. Math. Comput. Sci. 11 (2021), No. 2, 1486-1498. https://doi.org/10.28919/jmcs/5401 ISSN: 1927-5307.

[10]. Shehzad, N., Zeeshan, A., Ellahi, R., Vafai, K.: (2016) Convective heat transfer of nanofluid in a wavy channel: Buongiorno's mathematical model, J. Mol. Liq. 222 (2016) 446-455.

[11]. Mahanthesh, B., Mabood, F., Gireesha, B.J., Gorla, R.S.R.: (2017) Effects of chemical reaction and partial slip on the three-dimensional flow of a nanofluid impinging on an exponentially stretching surface, Eur. Phy. J. Plus 132 (2017) Article 113.

[12]. Kumar, K.G., Ramesh, G.K., Gireesha, B.J., Gorla, R.S.R.: (2018) Characteristics of joule heating and viscous dissipation on three-dimensional flow of Oldroyd B nanofluid with thermal radiation, Alexandria Eng. J. 57 (2018) 2139-2149.

[13]. Jiang, W., Chen, G.: (2019) Dispersion of active particles in confined unidirectional flows, J. Fluid Mech. 877 (2019) 1-34.

[14]. Xu, H.: (2019) Modelling unsteady mixed convection of a nanofluid suspended with multiple kinds of nanoparticles between two rotating disks by generalized hybrid model, Int. Commun. Heat Mass Transfer 108 (2019) 104275.

[15]. Alghamdi, M.: (2020) Significance of Arrhenius activation energy and binary chemical reaction in mixed convection flow of nanofluid due to a rotating disk, Coatings 10 (2020) Article 86.

[16]. Jiang, W., Chen, G.: (2020) Dispersion of gyrotactic micro-organisms in pipe flows, J. Fluid Mech. 889 (2020) Article 18.

[17]. Rafiq, T., Mustafa, M.: (2020) Computational analysis of unsteady swirling flow around a decelerating rotating porous disk in nanofluid, Arab. J. Sci. Eng. 45 (2020)1143-1154. 
[18]. Turkyilmazoglu, M.: (2020) Single phase nanofluids in fluid mechanics and their hydrodynamic linear stability analysis, Comput. Methods Prog. Biomed. 187 (2020)Article 105171.

[19]. Sadiq, M.A.: (2020) Serious solutions for unsteady axisymmetric flow over a rotating stretchable disk with deceleration, Symmetry 12 (2020) Article 96.

[20]. Niazi, M.D.K., Hang, X.: (2020) Modelling two-layer nanofluid flow in a micro-channel with electro-osmotic effects by means of Buongiorno's model, Appl. Math. Mech. 41(2020) 83-104.

[21]. Vimal Kumar Joshi., Dharmendra Tripathi., Paras Ram., Kushal Sharma.:(2017) Numerical investigation of magnetic nanofluids flow over rotating disk embedded in a porous medium. ResearchGate.

[22]. Abubakar, Nor- Ashikin., Bachok, Norfifah., Norihan, Md- Arifin., Ioan Pop.: (2018) Stability analysis on the flow and heat transfer of nanofluid past a stretching/shrinking cylinder with suction effect, Results in Physics 9 (2018) 1335-1344, https://doi.org/10.1016/j.rinp.2018.04.056

[23]. Fang, T., Zhang, J.: (2008) Flow between two stretchable disks-an exact solution of the Navier -Stokes equations. Int Commun Heat Mass Transf. 2008;35:89-2895.

[24]. Mushtaq. A., Mustafa. M.: (2017) Computations for nanofluid flow neaer a stretchable rotating disk with axial magnetic field and convective conditions. Result in physics 7 (2017) 3137-3144

[25]. ] Mustafa Turkyilmazoglu.: (2014) Nanofluid flow and heat transfer due to a rotating disk. Computers and fluid 94. 139-146

[26]. Noor Saeed Khan., Qayyum Shah., Amiya Bhaumik., Poom Kumam., Phatiphat Thounthong., Irajsadegh Amiri.:(2020) Entropy generation in bioconvection nanofluid flow between two stretchable rotating disks. Scientific Report (natureresearch).10:4448 | https://doi.org/10.1038/s41598-020-61172-2

[27]. Nor Ashikin Abu Bakara., Norfifah Bachokb., Norihan Md. Arifinb., Ioan Popc.: (2018) Stability analysis on the flow and heat transfer of nanofluid past a stretching/shrinking cylinder with suction effect. Results in Physics 9 (2018) 1335-1344. https://doi.org/10.1016/j.rinp.2018.04.056 